\title{
RELIABILITY OF A MECHANICAL SYSTEM WITH $K$-OUT-OF-N SUBSYSTEMS
}

\author{
KOKILA RAMESH ${ }^{1} \&$ RADHA GUPTA ${ }^{2}$ \\ ${ }^{1}$ Department of Mathematics, Faculty of Engineering and Technology, Jain (Deemed-to-be-University), Bangalore, India \\ ${ }^{2}$ Department of Mathematics, Dayananda Sagar College of Engineering, Bangalore, India
}

\begin{abstract}
Redundancy allocation problems having more general form for finding the optimal solutions has been demonstrated in the present paper. Different redundancy options offer enhanced capabilities in formulating the problem to accommodate k-out-of-n subsystems. Maximizing the system reliability given system-level constraints with the selection of the components and the redundancy levels is the objective. In view of this maximizing the system reliability having $k$-out-of-n subsystems becomes important with respect to cost and weight constraints. Determination of the system reliability by computing the stage reliabilities and the component reliabilities forms an integrated reliability model. Lagrange's method of undetermined multipliers is used in the present paper to find system reliability with the help of stage reliabilities and the component reliabilities. Also an attempt of using Random Search Technique for global optimization (RST) has been applied to find system and component reliabilities for comparison.

KEYWORDS: Redundancy Allocation, k-out-of-n Configuration, Lagrangian Multiplier, Random Search Technique (RST)
\end{abstract}

Received: Jun 06, 2020; Accepted: Jun 26, 2020; Published: Aug 07, 2020; Paper Id.: IJMPERDJUN2020679

\section{INTRODUCTION}

In many complex systems it is difficult to improve the system reliability by just selecting the good quality components only. It is desired to have a redundant and backup elements and subsystems to improve or enhance the total system reliability. Such a special case in parallel redundancy is $k$-out-of- $n$ configuration. Out of the total $n$ parallel components at least $k$ components should succeed for the system to succeed in this type of system configuration. In this paper to increase the reliability of the mechanical system with the constraints given, the redundancy allocation involving the selection of the type $k$-out-of- $n$ configuration is used. Here the direct relationship between cost and weight and component reliabilities has been highlighted to compute system reliability.

There is a continued research activity in optimizing the redundancy allocation problems of different types. Barlow and Heidtmann 1984 were the earliest members proposing the reliability computations for system having $k$ out-of- $n$ subsystems. Related to the above authors work, generalizing the subject and application on the problems was carried out by Jain and Gopal 1985, Risse 1987 and Sarje and Prasad 1989 and many others. Chiang and Niu 1981 introduced the work on consecutive k-out-of-n systems and Hwang 1982, Derman et al 1982, Shanthikumar 1982, Ge and Wang 1990 proposed the efficient algorithms for the system reliability effectively. Yamamoto and Miyakawa 1995 have developed exact reliability finding models and Koutras et al. 1997 have introduced bounds for rectangular two-dimensional consecutive models. Pham 1992 described an optimal design to optimize problems related to $k$-out-of- $n$ subsystems which are redundant in order to minimize the total cost and also to find the number of units which is optimal for a system consisting of only one k-out-of-n subsystem. Further, Pham 1996 
demonstrated different techniques to determine the number of components in $k$-out-of- $n$ subsystems which is optimal, most economical and also to minimize the mean total cost of the system. Suich and Patterson 1991 described a solution which is numerical for $k$ and $n$, which also minimized the cost of the system having $k$-out-of- $n$ subsystems and derived different cost minimization models having single k-out-of- $n$ subsystem. Pham and Malon 1994 achieved in finding the optimal system size $n$ and a threshold $k$ for $k$-out-of- $n$ subsystem problem using failure modes of competent nature. Chiang and Chiang studied the method of choosing an optimal $k$ in $k$-out-of- $n$ system with no closed form solution. The work of Huang et al 2000 and Z. Tian et al 2009 shows variable $k$ on multi-state $k$-out-of-n system with different states of the system with number of components required being dynamic. M. Zuo and Z. Tian 2006 proposed a more generalized multi-state $k$-outof- $n$ systems and its performance evaluation to expand the model of Huang et al 2000 to generalized multi-state $k$-out-of$n:$ F system from generalized multi-state $k$-out-of- $n$ :G system.

Huang et al 2000 made an effort to modify the required number of components of multiple states in each state for practical applications, where as David W. Coit et al 2012 proposed a subsystem having non identical mixing of components with a binary state under condition being the dynamic required number of components for each working subsystem which may be suitable for the possible application on the problem. S. V. Suresh Babu et al 2012 worked on the multiple constraints problem first solved using the method of Lagrangean multipliers providing a real valued solution which may be infeasible for practical implementation for finding reliability of the system. This problem was solved by Dynamic Programming in taking the number of components as integer values for proving it to be ideal solution and to find the exact system reliability. In continuation with this system reliability with cost and weight constraints is analyzed with respect to component reliability in the form of sensitivity analysis. Further in the work of Sridhar et al 2013, an attempt has been made to maximize the reliability of the $k$-out-of- $n$ system using redundant integrated reliability model with multiple constraints. In this work Lagrangian approach of solving the reliability system has been considered using dynamic programming. Also the effort has been made by the authors K.S. Lakshminarayana and Y. Vijaya Kumar 2013 for the given mathematical function to establish the results by using Lagrangian multiplier method, but these results were in real values of number of components required, which was rounded off to the next integer value resulting in major variation in all the constraints. But it was seen that the dynamic Programming results in very little variation in all the constraints. Hence a critical analysis was made to identify and correct the causes with the help of failure modes effects and also to compliment the results obtained through the dynamic programming.

A different method is demonstrated and presented in the present paper to determine solutions which are optimal to the problem having redundancy allocation which is the more general form of the system reliability. The problem is solved by a method called Random Search Technique (RST) method which gives an approximate solution. Also tries to give a comparison to this results using Lagrangian approach of undetermined multipliers using MATLAB software. Maximization of the system reliability subjected to cost and a weight constraint is the main objective of the present work with $k$-out-of- $n$ series configuration. Maximization of system reliability with $k$-out-of- $n$ series configuration can be done in two different ways. 1. Maximizing the system reliability by determining the number of components in each stage with the known component reliabilities for the given cost and weight constraints. 2. Determination of the component reliabilities with the known number of components to maximize system reliability for the given cost and weight constraints. 


\section{MATHEMATICAL MODEL}

The reliability models with the objective function having the constraints are:

$$
R_{S}=\prod_{i=1}^{n} R_{i}, R_{i}=\sum_{k=2}^{x_{i}}\left(\begin{array}{c}
x_{i} \\
k
\end{array}\right) r_{i}^{k}\left(1-r_{i}\right)^{x_{i}-k}
$$

Subject to :

$$
\begin{gathered}
\sum_{i=1}^{n} c_{i} x_{i} \leq C_{0} \\
\sum_{i=1}^{n} w_{i} x_{i} \leq W_{0} \\
x_{i} \geq 0 \quad \forall i
\end{gathered}
$$

Where $R_{s}$-System reliability, $R_{i}$-Stage reliability, $r_{i}$-Component reliability, $x_{i}$-Number of components in stage i is assumed to be constant, $c_{i}$-coefficient of the cost of each component in $\mathrm{i}^{\text {th }}$ stage, $w_{i}$-coefficient of the weight of each component in $\mathrm{i}^{\text {th }}$ stage, $C_{o}$-Maximum cost allowable for the system, $W_{o}$-Maximum weight allowable for the system.

\section{PROBLEM FORMULATION}

The problems related to reliability optimization in the literature have the decision variables as the number of redundancies being integers and the component reliabilities with real numbers or a combination of both. This method uses the differentiation of the decision variables which have to be continuous. In this paper the formulation of the integrated reliability models under the specified mathematical function is considered with ' $\mathrm{i}$ ' stages being independent statistically in series with $x_{i}$ components being independent statistically in each stage.

Case (i): Coefficient of cost of every component in $\mathrm{i}^{\text {th }}$ stage is derived using the relationship between cost and reliability given by $r_{i}=\left[c_{i} / a_{i}\right]^{\left(1 / b_{i}\right)}$ i.e, $c_{i}=a_{i} \cdot r_{i}^{b_{i}}$ cost constraint. Similarly using the same relationship between the reliability and weight is given by $w_{i}=e_{i} \cdot r_{i}^{f_{i}}$ weight constraint. Since cost constraint is linear in $x_{i}$, substituting the above relations in the mathematical model constraints, the following equations are obtained

$$
\sum_{i=1}^{n}\left[\left(a_{i} \cdot r_{i}^{b_{i}}\right) x_{i}\right]-C_{0} \leq 0 \text { and } \sum_{i=1}^{n}\left[\left(e_{i} \cdot r_{i}^{f_{i}}\right) x_{i}\right]-W_{0} \leq 0
$$

Hence the model becomes

$$
\begin{aligned}
\text { Maximize } R_{s}= & \prod_{i=1}^{n} \sum_{k=2}^{x_{i}}\left(\begin{array}{c}
x_{i} \\
k
\end{array}\right) r_{i}^{k}\left(1-r_{i}\right)^{x_{i}-k} \\
\text { Subject to } & \sum_{i=1}^{n}\left[\left(a_{i} \cdot r_{i}^{b_{i}}\right) x_{i}\right]-C_{0} \leq 0 \\
& \sum_{i=1}^{n}\left[\left(e_{i} \cdot r_{i}^{f_{i}}\right) x_{i}\right]-W_{0} \leq 0
\end{aligned}
$$


Non-negativity restriction $r_{i} \geq 0$

Case (ii): Coefficient of the cost of every component in $\mathrm{i}^{\text {th }}$ stage is derived from the relationship between cost and reliability given by $c_{i}=a_{i} \exp \left[\frac{b_{i}}{\left(1-r_{i}\right)}\right]$, cost constraint. Similarly using the same relationship between the reliability and weight is given by $w_{i}=e_{i} \exp \left[\frac{f_{i}}{\left(1-r_{i}\right)}\right]$, weight constraint. Substituting the above relations in the mathematical model, the following results are obtained

$$
\begin{gathered}
\sum_{i=1}^{n}\left[\left(a_{i} \exp \left[\frac{b_{i}}{\left(1-r_{i}\right)}\right]\right) \cdot x_{i}\right]-C_{0} \leq 0 \\
\sum_{i=1}^{n}\left[\left(e_{i} \exp \left[\frac{f_{i}}{\left(1-r_{i}\right)}\right]\right) \cdot x_{i}\right]-W_{0} \leq 0
\end{gathered}
$$

Hence the model becomes

$$
\begin{aligned}
\text { Maximize } & R_{s}=\prod_{i=1}^{n} \sum_{k=2}^{x_{i}}\left(\begin{array}{c}
x_{i} \\
k
\end{array}\right) r_{i}^{k}\left(1-r_{i}\right)^{x_{i}-k} \\
\text { Subject to } & \sum_{i=1}^{n}\left[\left(a_{i} \exp \left[\frac{b_{i}}{\left(1-r_{i}\right)}\right]\right) \cdot x_{i}\right]-C_{0} \leq 0 \\
& \sum_{i=1}^{n}\left[\left(e_{i} \exp \left[\frac{f_{i}}{\left(1-r_{i}\right)}\right]\right) \cdot x_{i}\right]-W_{0} \leq 0
\end{aligned}
$$

Non-negativity restriction $r_{i} \geq 0$

\section{METHODOLOGY}

Case (i): A Lagrangian function is formulated as

$$
F=R_{s}+\lambda_{1}\left[\sum_{i=1}^{n}\left\{\left(a_{i} r_{i}^{b_{i}}\right) x_{i}\right\}-C_{o}\right]+\lambda_{2}\left[\sum_{i=1}^{n}\left\{\left(e_{i} r_{i}^{f_{i}}\right) x_{i}\right\}-W_{o}\right] \text { Where } \lambda_{1} \text { and } \lambda_{2} \text { are undetermined multipliers }
$$

The Lagrangian function is differentiated with respect to $r_{i}, \lambda_{1}$ and $\lambda_{2}$ to obtain the stationary points as follows

$$
\begin{gathered}
\frac{\partial F}{\partial r_{i}}=\lambda_{1}\left[\left\{\sum_{i=1}^{n} b_{i} a_{i} r_{i}^{b_{i}-1}\right\} x_{i}\right]+\lambda_{2}\left[\left\{\sum_{i=1}^{n} f_{i} e_{i} r_{i}^{f_{i}-1}\right\} x_{i}\right]=0 \\
\frac{\partial F}{\partial \lambda_{1}}=\left[\left\{\sum_{i=1}^{n} a_{i} r_{i}^{b_{i}}\right\} x_{i}\right]-C_{o}=0 \text { and } \frac{\partial F}{\partial \lambda_{2}}=\left[\left\{\sum_{i=1}^{n} e_{i} r_{i}^{f_{i}}\right\} x_{i}\right]-W_{o}=0
\end{gathered}
$$


Table 1: The Data Required for Cost and Weight Constants for Finding System Reliability

\begin{tabular}{|c|c|c|c|c|}
\hline \multirow{2}{*}{ STAGE (i ) } & \multicolumn{2}{|c|}{ Cost Constants } & \multicolumn{2}{c|}{ Weight Constants } \\
\cline { 2 - 5 } & $a_{i}$ & $b_{i}$ & $e_{i}$ & $f_{i}$ \\
\hline 1 & 25 & 2 & 25 & 2 \\
\hline 2 & 75 & 3 & 150 & 3 \\
\hline 3 & 55 & 4 & 155 & 4 \\
\hline
\end{tabular}

Case (ii): Lagrangean Method for the function $c_{i}=a_{i} \exp \left[\frac{b_{i}}{\left(1-r_{i}\right)}\right]$. A Lagrangean function is formulated as:

$$
\begin{gathered}
F=R_{s}+\lambda_{1}\left[\sum_{i=1}^{n} F_{1}-C_{o}\right]+\lambda_{2}\left[\sum_{i=1}^{n} F_{2}-W_{o}\right] \\
\text { Where } F_{1}=\left\{\left(a_{i} \exp \left[\frac{b_{i}}{\left(1-r_{i}\right)}\right]\right) x_{i}\right\} \text { and } F_{2}=\left\{\left(e_{i} \exp \left[\frac{f_{i}}{\left(1-r_{i}\right)}\right]\right) x_{i}\right\}
\end{gathered}
$$

Where $\lambda_{1}$ and $\lambda_{2}$ are undetermined multipliers

The Lagrangian function is differentiated with respect to $r_{i}, \lambda_{1}$ and $\lambda_{2}$ to obtain the stationary points as follows

$$
\begin{gathered}
\frac{\partial F}{\partial r_{i}}=\lambda_{1} F_{3}+\lambda_{2} F_{4}=0 \\
\text { Where } F_{3}=\left[\left\{\sum_{i=1}^{n} \frac{a_{i} b_{i}}{\left(1-r_{i}\right)^{2}} \exp \left[\frac{b_{i}}{\left(1-r_{i}\right)}\right]\right\} x_{i}\right] \text { and } F_{4}=\left[\left\{\sum_{i=1}^{n} \frac{e_{i} f_{i}}{\left(1-r_{i}\right)^{2}} \exp \left[\frac{f_{i}}{\left(1-r_{i}\right)}\right]\right\} x_{i}\right] \\
\frac{\partial F}{\partial \lambda_{1}}=\left[\left\{\sum_{i=1}^{n} a_{i} \exp \left[\frac{b_{i}}{\left(1-r_{i}\right)}\right]\right\} x_{i}\right]-C_{o}=0 \text { and } \frac{\partial F}{\partial \lambda_{2}}=\left[\left\{\sum_{i=1}^{n} e_{i} \exp \left[\frac{f_{i}}{\left(1-r_{i}\right)}\right]\right\} x_{i}\right]-W_{o}=0
\end{gathered}
$$

Table 2: The Data Required for Cost and Weight Constants for Finding System Reliability

\begin{tabular}{|c|c|c|c|c|}
\hline \multirow{2}{*}{ STAGE (i ) } & \multicolumn{2}{|c|}{ Cost Constants } & \multicolumn{2}{c|}{ Weight Constants } \\
\cline { 2 - 5 } & $a_{i}$ & $b_{i}$ & $e_{i}$ & $f_{i}$ \\
\hline 1 & 100 & 0.7 & 100 & 0.9 \\
\hline 2 & 120 & 0.8 & 150 & 0.8 \\
\hline 3 & 94 & 0.5 & 110 & 0.7 \\
\hline
\end{tabular}

\section{NUMERICAL RESULTS}

The present work is to maximize the mechanical system reliability with $k$-out-of- $n$ subsystems as objective. To maximize the reliability of the problem, a mathematical relation between the component reliabilities and the constraints (cost and weight) are used. The solution using the Lagrangian method is obtained with the help of MATLAB toolbox, which gives a unique solution to the problem. This method is an analytical way of solving the problem. Also another methodology applied for the comparison is controlled RST for global optimization. The component reliabilities, stage reliabilities and the system reliability are determined in each case. In the present methodology, number of components is assumed to be constant and only three stages are considered due to complexity of the problem. 
Case (i): In the case of a mechanical system with number of stages being three and the number of components assumed to be five, the relation assumed between cost and the component reliability is $r_{j}=\left[\frac{c_{j}}{a_{j}}\right]^{\frac{1}{b_{j}}}$. The system cost of Rs 250 and system weight $400 \mathrm{kgs}$ is assumed to be maximum values to determine component reliability $\left(r_{i}\right)$, stage reliability $\left(R_{i}\right)$ and the system reliability $\left(R_{s}\right)$ which is optimum.

Results Obtained using Lagrangian Multipliers Method

Table 3: In this Table the System Reliability Related to Cost and Weight of each Component with its Reliability is shown for Case (i) Relation

\begin{tabular}{|c|c|c|c|c|c|c|c|}
\hline STAGE $(\mathrm{i})$ & $r_{i}$ & $R_{i}$ & $x_{i}$ & $c_{i}$ & $w_{i}$ & $x_{i} c_{i}$ & $x_{i} w_{i}$ \\
\hline 1 & 0.9762 & 0.99 & 5 & 23.82 & 23.82 & 119 & 119 \\
\hline 2 & 0.6593 & 0.95 & 5 & 21.49 & 42.99 & 108 & 215 \\
\hline 3 & 0.5400 & 0.86 & 5 & 4.68 & 13.18 & 23 & 66 \\
\hline \\
TOTAL COST AND WEIGHT & 250 & 400 \\
\hline
\end{tabular}

\section{Results Obtained using Controlled RST}

Controlled random search technique is a global optimization technique. This gives a flexibility of using a range of values for the variables to search and optimize. This methodology works well for the non-linear problems. The results of the problem are obtained by the RST and are given in Table 4.

Table 4: In this Table the System Reliability Related to Cost and Weight of each Component with its Reliability is Shown for Case (i) Relation in Ten Runs of the RST Algorithm

\begin{tabular}{|c|c|c|c|c|c|c|c|c|c|c|}
\hline Run No & 1 & 2 & 3 & 4 & 5 & 6 & 7 & 8 & 9 & 10 \\
\hline \multirow{3}{*}{$r_{i}$} & 0.97 & 0.87 & 0.87 & 0.94 & 0.97 & 0.88 & 0.89 & 0.89 & 0.92 & 0.97 \\
\hline & 0.65 & 0.66 & 0.65 & 0.66 & 0.65 & 0.66 & 0.66 & 0.65 & 0.66 & 0.65 \\
\hline & 0.55 & 0.55 & 0.54 & 0.55 & 0.55 & 0.54 & 0.55 & 0.55 & 0.55 & 0.55 \\
\hline \multirow{3}{*}{$R_{i}$} & 0.99 & 0.99 & 0.99 & 0.99 & 0.99 & 0.99 & 0.99 & 0.99 & 0.99 & 0.99 \\
\hline & 0.95 & 0.95 & 0.94 & 0.95 & 0.95 & 0.95 & 0.95 & 0.94 & 0.95 & 0.95 \\
\hline & 0.86 & 0.86 & 0.85 & 0.86 & 0.86 & 0.85 & 0.86 & 0.86 & 0.86 & 0.86 \\
\hline$x_{i}$ & 5 & 5 & 5 & 5 & 5 & 5 & 5 & 5 & 5 & 5 \\
\hline \multirow{3}{*}{$c_{i}$} & 23.5 & 18.9 & 08.9 & 22.1 & 23.5 & 19.4 & 19.8 & 19.8 & 21.2 & 23.5 \\
\hline & 21.6 & 21.6 & 20.6 & 21.6 & 21.6 & 21.6 & 21.6 & 20.6 & 21.6 & 21.6 \\
\hline & 5.03 & 5.03 & 4.68 & 5.03 & 5.03 & 4.68 & 5.03 & 5.03 & 5.03 & 5.03 \\
\hline \multirow{3}{*}{$x_{i} c_{i}$} & 118 & 95 & 95 & 111 & 118 & 97 & 99 & 99 & 106 & 118 \\
\hline & 107 & 107 & 102 & 107 & 107 & 107 & 107 & 103 & 107 & 107 \\
\hline & 25 & 25 & 25 & 25 & 25 & 25 & 25 & 25 & 25 & 25 \\
\hline Total Cost & 250 & 227 & 220 & 243 & 250 & 227 & 231 & 227 & 238 & 250 \\
\hline \multirow{3}{*}{$w_{i}$} & 23.5 & 18.9 & 18.9 & 22.1 & 23.5 & 19.4 & 19.8 & 19.8 & 21.2 & 23.5 \\
\hline & 43.12 & 43.12 & 41.19 & 43.12 & 43.12 & 43.12 & 43.12 & 41.19 & 43.12 & 43.12 \\
\hline & 14.18 & 14.18 & 13.17 & 14.18 & 14.18 & 13.18 & 14.18 & 14.18 & 14.18 & 14.18 \\
\hline \multirow{3}{*}{$x_{i} w_{i}$} & 116 & 95 & 95 & 111 & 116 & 96 & 100 & 100 & 106 & 116 \\
\hline & 214 & 216 & 206 & 216 & 214 & 215 & 216 & 206 & 216 & 214 \\
\hline & 70 & 70 & 66 & 70 & 70 & 66 & 70 & 70 & 70 & 70 \\
\hline $\begin{array}{c}\text { Total } \\
\text { Weight }\end{array}$ & 400 & 381 & 367 & 397 & 400 & 378 & 386 & 376 & 392 & 400 \\
\hline$R_{S}$ & 0.827 & 0.826 & 0.811 & 0.827 & 0.827 & 0.816 & 0.826 & 0.821 & 0.826 & 0.827 \\
\hline
\end{tabular}


Case (ii): Consider the case of a mechanical system with three stages and the number of components assumed to be five for which the component reliability is given by the equation $c_{i}=a_{i} \exp \left[\frac{b_{i}}{\left(1-r_{i}\right)}\right]$. The system cost of Rs 5000 and system weight $7500 \mathrm{kgs}$ are assumed to be maximum values to determine the optimum Component reliability $\left(r_{i}\right)$, Stage reliability $\left(R_{i}\right)$ and the System reliability $\left(R_{s}\right)$.

\section{Results Obtained using Lagrangian Method}

Table 5: In this Table the System Reliability Related to Cost and Weight of Each Component with its Reliability is Shown for Case (ii) Relation

\begin{tabular}{|c|c|c|c|c|c|c|c|}
\hline STAGE (i) & $r_{i}$ & $R_{i}$ & $x_{i}$ & $c_{i}$ & $w_{i}$ & $x_{i} c_{i}$ & $x_{i} w_{i}$ \\
\hline 1 & 0.50 & 0.91 & 5 & 405.5 & 604.9 & 2028 & 3025 \\
\hline 2 & 0.47 & 0.90 & 5 & 542.9 & 678.6 & 2714 & 3393 \\
\hline 3 & 0.56 & 0.95 & 5 & 292.85 & 539.9 & 1464 & 2700 \\
\hline \multicolumn{6}{|c|}{ TOTAL COST AND TOTAL WEIGHT } & \multicolumn{3}{|c|}{$\mathbf{0 . 7 8}$} \\
\hline PERCENTAGE VARIATION IN THE COST AND & $\mathbf{2 4 \%}$ & $\mathbf{2 1 . 5 \%}$ \\
\hline
\end{tabular}

\section{Results Obtained using Controlled RST}

The result of the problem is obtained by the controlled RST and is given in Table 6 . The percentage of variance in the cost and the weight is given in Table 7

Table 6: In this Table the System Reliability Related to Cost and Weight of each Component with its Reliability is Shown for Case (ii) Relation in Ten Runs of the RST Algorithm

\begin{tabular}{|c|c|c|c|c|c|c|c|c|c|c|}
\hline Run No & 1 & 2 & 3 & 4 & 5 & 6 & 7 & 8 & 9 & 10 \\
\hline \multirow[t]{3}{*}{ 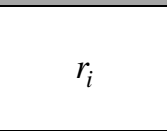 } & 0.48 & 0.49 & 0.48 & 0.49 & 0.49 & 0.49 & 0.50 & 0.49 & 0.49 & 0.48 \\
\hline & 0.49 & 0.49 & 0.49 & 0.49 & 0.46 & 0.50 & 0.48 & 0.48 & 0.48 & 0.48 \\
\hline & 0.56 & 0.58 & 0.57 & 0.56 & 0.59 & 0.57 & 0.59 & 0.59 & 0.58 & 0.57 \\
\hline \multirow{3}{*}{$R_{i}$} & 0.89 & 0.91 & 0.89 & 0.91 & 0.91 & 0.91 & 0.94 & 0.91 & 0.91 & 0.89 \\
\hline & 0.91 & 0.91 & 0.91 & 0.91 & 0.84 & 0.94 & 0.89 & 0.89 & 0.89 & 0.89 \\
\hline & 0.95 & 0.99 & 0.96 & 0.95 & 0.99 & 0.96 & 0.99 & 0.99 & 0.99 & 0.95 \\
\hline$x_{i}$ & 5 & 5 & 5 & 5 & 5 & 5 & 5 & 5 & 5 & 5 \\
\hline \multirow{3}{*}{$c_{i}$} & 384.26 & 394.54 & 384.26 & 394.54 & 394.54 & 394.54 & 405.52 & 394.54 & 394.54 & 384.26 \\
\hline & 576 & 576 & 576 & 576 & 528 & 594 & 559 & 559 & 559 & 559 \\
\hline & 292.85 & 309.13 & 300.69 & 292.85 & 318.24 & 300.69 & 318.24 & 318.24 & 309.13 & 300.69 \\
\hline \multirow{3}{*}{$c_{i} x_{i}$} & 1921 & 1973 & 1921 & 1973 & 1973 & 1973 & 2028 & 1973 & 1973 & 1921 \\
\hline & 2880 & 2880 & 2880 & 2880 & 2640 & 2972 & 2795 & 2795 & 2795 & 2795 \\
\hline & 1464 & 1546 & 1504 & 1464 & 1591 & 1504 & 1591 & 1591 & 1546 & 1504 \\
\hline Total Cost & 6265 & 6399 & 6305 & 6317 & 6204 & 6449 & 6414 & 6359 & 6314 & 6220 \\
\hline \multirow{3}{*}{$w_{i}$} & 564.50 & 583.99 & 564.50 & 583.99 & 583.99 & 583.99 & 604.97 & 583.99 & 583.99 & 564.50 \\
\hline & 720.00 & 720.00 & 720.00 & 720.00 & 659.92 & 742.96 & 698.61 & 698.61 & 698.61 & 698.61 \\
\hline & 539.90 & 582.39 & 560.25 & 539.90 & 606.56 & 560.25 & 606.56 & 606.56 & 582.39 & 560.25 \\
\hline \multirow{3}{*}{$w_{i} x_{i}$} & 2823 & 2920 & 2823 & 2920 & 2920 & 2920 & 3025 & 2920 & 2920 & 2823 \\
\hline & 3600 & 3600 & 3600 & 3600 & 3299 & 3715 & 3493 & 3493 & 3493 & 3493 \\
\hline & 2699 & 2912 & 2801 & 2699 & 3033 & 2801 & 3033 & 3033 & 2912 & 2801 \\
\hline $\begin{array}{c}\text { Total } \\
\text { Weight }\end{array}$ & 9122 & 9432 & 9224 & 9219 & 9252 & 9436 & 9551 & 9446 & 9325 & 9117 \\
\hline$R_{S}$ & 0.8911 & 0.9658 & 0.9151 & 0.9154 & 0.9141 & 0.9657 & 0.9920 & 0.9658 & 0.9400 & 0.8909 \\
\hline
\end{tabular}


Table 7: Percentage of Variance in the Cost and Weight

\begin{tabular}{|c|c|c|c|c|c|c|c|c|c|c|}
\hline Runs & $\mathbf{1}$ & $\mathbf{2}$ & $\mathbf{3}$ & $\mathbf{4}$ & $\mathbf{5}$ & $\mathbf{6}$ & $\mathbf{7}$ & $\mathbf{8}$ & $\mathbf{9}$ & $\mathbf{1 0}$ \\
\hline $\begin{array}{c}\text { \% Variance } \\
\text { in Cost }\end{array}$ & 25.30 & 27.98 & 26.10 & 26.34 & 24.08 & 28.98 & 28.28 & 27.18 & 26.28 & 24.40 \\
\hline $\begin{array}{c}\text { \% Variance } \\
\text { in Weight }\end{array}$ & 21.63 & 25.76 & 22.99 & 22.92 & 23.36 & 25.81 & 27.35 & 25.95 & 24.33 & 21.56 \\
\hline
\end{tabular}

\section{DISCUSION}

Optimizing reliability of a mechanical system with $k$-out-of- $n$ subsystems has always been a complex system to study. An attempt has been made in the present paper to study this complex system using the transformations given in the above section. It has been noticed that using Lagrange's method of undetermined multipliers with the help of MATLAB toolbox, the system reliability obtained for the mechanical system in case (i) is 0.81 and in case (ii) is 0.78 . There is a scope of improving this model by using different mathematical relations between the component reliabilities and the constraints. To compare with the existing method, RST has been applied and the average system reliability of ten runs in case (i) and case (ii) are 0.82 and 0.89 respectively. Total cost and Total weight in each run is either less than or equal to maximum allowable cost and weight in both the methods. The flexibility in the RST helps us to have a range of values for the component, stage and system reliabilities. There is no much variation in the maximum allowable cost and weight in both the approaches used to maximize the system reliability. From the above discussion it is clear that the results obtained are optimal and the matches with the cost and the weight constraints.

\section{CONCLUSIONS}

An Integrated Model for finding reliability with $k$-out-of- $n$ subsystems having multiple constraints is established while optimizing the system reliability using mathematical function. The reliability model refers to determination of System Reliability $\left(R_{s}\right)$, Stage Reliability $\left(R_{j}\right)$ and the Component Reliabilities $\left(r_{j}\right)$. These values are calculated using Lagrangian Multiplier Approach, which is an analytical method and gives a unique solution satisfying the constraints. As the complexity of the problem increases, the methodology of solving using Lagrangian method becomes cumbersome. Hence Controlled Random Search Technique has been used as an alternative method which provides many possible solution sets to maximize the system reliability of a mechanical system without compromising on its quality. Further, we have applied the same method for maximizing the system reliability of a mechanical system with cost and the weight constraints and ten runs were sufficient to optimize the system reliability. Hence it is concluded that the techniques used are effective in solving non-linear optimization problem, specifically in finding reliability of a mechanical system having $k$-out-of- $n$ subsystems. Further, in the present reliability model number of components is assumed in each stage. If we consider the number of components to be variable, sufficient research work can be established. Also in the case of $k$-out-of-n subsystems, both $k$ and $n$ can be assumed to be variables. The scope of this kind of models will be of great importance.

\section{REFERENCES}

1. Chiang, D.T. and Niu, S.C. (1981), “Reliability of a consecutive-k-out-of-n: F system”, IEEE Transaction on Reliability, 30: 87-89.

2. Derman. D., Lieberman. G and Ross S. (1982), “On the consecutive-k-out-of-n: F system”, IEEE Trans. Reliability 31, 57-63.

3. Hwang, F. K. (1982), “Fast Solutions for Consecutive-k-out-of-n:F System”, IEEE Transactions on Reliability, Vol. R-31, No. 5, $p$ p 447-448. 
4. Shantikumar, J.G., (1982), "Recursive algorithm to evaluate the reliability of consecutive k-out-of-n:F system networks", IEEE Trans. Reliab., 31: 84-87.

5. Barlow, R.E. and Heidtmann, K.D. (1984) “Computing k-out-of-n system

6. Reliability”, IEEE Transactions on Reliability, 33, 322-323.

7. S. P. Jain and K. Gopal (1985) "Reliability of k-to-l-out-of-n systems”, Reliability Engineering, 12:175-179.

8. T Risse. (1987) "On the evaluation of the reliability of k-out-of-n systems", IEEE Transactions on Reliability, 224-234.

9. Silvestri, Marco, T. I. Z. I. A. N. O. Cucchi, and Matteo Confalonieri. "An Innovative Electric Ship Steering System: Analysis and Implementation." International Journal of Mechanical and Production Engineering Research and Development 9.4 (2019): 415-424.

10. Sarje. A. K and E. V. Prasad (1989), "An efficient Non-recursive algorithm for computing the reliability of k-out-of-n systems", IEEE Trans. on Reliability, Vol. R-38, No-2, pp-234-235.

11. G. Ge and L. Wang (1990), "Exact Reliability Formula for Consecutive-k-out-of-n:F Systems with Homogeneous Markov Dependence”, IEEE Trans. On Reliability, Vol. 39, No. 5.

12. Hoang Pham (1992), "Optimal Design of k-out-of-n Redundant Systems”, Microelectronics and Reliability 32(1/2), pp. 119126.

13. A. Rushdi and K. A. AI-Hindi (1993), "Table of the Lower Boundary of the Region of Useful Redundancy for k-out-of-n System”, Microelectronics and Reliability 33(7), pp. 979-992.

14. Hoang Pham and D. M. Malon (1994), “Optimal design of systems with competing failure modes", IEEE Trans. Reliab. 43(2), pp. 251-254.

15. H.Yamamoto and M. Miyakawa (1995), "Reliability of a circular connected -(r,s)-out-of-(m,n):F Lattice system”, Journal of the Operations Research, Vol. 39, No. 3.

16. Kumar, Praveen, et al. "A Study on HR Audit in Organization Systems of BHEL Hyderabad." International Journal of Business and General Management 4.5 (2015): 49-58.

17. Hoang Pham (1996), “On the Optimal Design of k-out-of-n:G Subsystems”, IEEE Trans. Reliab. 45(2), pp. 254-260.

18. Koutras, M. V (1997), “Consecutive k,r-out-of-n:DFM systems”, Microelectronics and Reliability, 37, 597-603.

19. J. Huang, M. J. Zuo and Y. H. Wu (2000), “Generalized multi-state k-out-of-n:G system”, IEEE Trans Reliab, Vol. No. 1 pp $105-111$.

20. Singh, Ajay, and Shakti Kumar. "Reliability Optimization Using Hardware Redundancy: A Parallel BB-BC Based Approach." International Journal of Electronics and Communication Engineering (IJECE) ISSN (P) (2015): 2278-9901.

21. Chen, Y. and Yang Q. (2005), "Reliability of two-stage weighted k-out-of-n systems with components in common", IEEE Transactions on Reliability, 54, no. 3, 431-440.

22. Aru, Suraj, et al. "Design, analysis and optimization of a multi-tubular space frame." International Journal of Mechanical and Production Engineering Research and Development (IJMPERD) ISSN (P) (2014): 2249-6890.

23. M. Zuo and Z. Tian (2006), "Performance Evaluation of Generalized Multi-State k-out-of-n Systems", Rel, IEEE Trans on V. 55, Issue: 2.

24. H. Taha, Integer Programming: Theory, Applications, and Computations (Academic Press, 1975). 
25. S. V. Suresh Babu, D.Maheswar, G. Ranganath, Y.Vijaya Kumar and G.Sankaraiah (2012), “ Redundancy Allocation for Series Parallel Systems with Multiple Constraints and Sensitivity Analysis”, IOSR Journal of Engineering, Vol-2(3), pp-424428.

26. A.Sridhar, S.Pavan Kumar, Y.Raghunatha Reddy, G.Sankaraiah and C.Umashankar (2013), "The k-out-of-n Redundant IRM Optimization with multiple constraints" IJRSAT, Issue-2, Vol-1.

27. K. S. Lakshminarayana and Y. Vijay Kumar (2013), "Reliability optimization of integrated reliability model using dynamic programming and failure modes effects and criticality analysis”, J. Acad. Indus. Res. Vol. 1(10), pp-622-625. 